\title{
MET OLSR - An Energy Effective OLSR based Routing Protocol
}

\author{
Ankita Khapre \\ M. Tech Scholar \\ NIIST Bhopal MP
}

\author{
Umesh Lilhore \\ Assistant Prof. \\ NIIST Bhopal MP
}

\begin{abstract}
WSN today is an emerging field of research where different routing protocol takes part in order to compute the routing facility and data transfer in between various available nodes and medium. OLSR is one of the advantageous protocol while dealing with energy and efficient resource utilization scenario thus it is a growing area today. We have already discussed about the different available technique in our literature paper where the modification is still require when the nodes numbers are increased and energy level need to further optimized while communication in modified TC packets. TC packet leads to carry the information of weight and node information which means to TC packet modification scenario, thus the protocol is proposed MET OLSR technique which is in terms to store the state and weight of the nodes after each communication and thus it make available for next computation. In this paper we discuss about the proposed methodology MET OLSR and further we compute the comparison analysis in between existing OLSR and MET OLSR in terms of PDR and other relevant parameters.
\end{abstract}

\section{Keywords}

TC Packets, OLSR, PDR, MET, WSN, NRL.

\section{INTRODUCTION}

Wireless sensor networks are the network where multiple sensor nodes are connected to transmit data from source to destination. In wireless sensor network scenario small micro sensor nodes are used to communicate one another but route message in a continuous changing scenario. Fine an optimize route in WSN is an combinatorial problem thus a new t5echnique is required to select optimized path from the set of path.

Find optimal route to Route packets in wireless sensor networks is one of the major issue in wireless sensor network. Because movable nodes are used in such network, thus topology of the networks changes frequently. Thus find an optimal path to route packets is a challenging task to do. There are various meta-heuristic algorithms are presented to provide better solution to find optimal route in wireless sensor network scenario

A new technique called MET -OLSR (Modified Energy Threshold Based OLSR) is proposed in this dissertation, which provides enhanced functionality to transmit packets in wireless sensor network scenario. A weight assigning mechanism is used to track the optimize path by detecting modification in optimize paths. Transmission control packets are used to track the process of path optimization from different nodes. A security mechanism is also provided which enhances the security of the whole technique.

\section{RELATED WORK}

In our recent paper the technique OLSR is introduced where a routing matrix introduced along with the existing standard
OLSR with which the following concepts were added for reducing energy consumption at each node to get maximum output from a network in wireless sensor network communication. Packet transmission and Overhearing from the neighbor nodes are two important factors which consider for the energy efficient routing according to their work. They have utilized three routing approach namely [1] 1. MAC queue utilization 2. Residual energy, 3. Node degree. They have changed the proactive manner of standard OLSR and TC packet format in order to make integrated routing scheme. They have used Greedy Heuristic Algorithm for the routing table computation for efficient routing scheme in enhanced OLSR work done by them. And in future they kept a work in large network to make a network system fast due to their changes done in TC packets. Hence robustness of system can be done by proceeding the work.

In the field of WSN for increasing the network lifetime by reducing energy usability in the network routing scenario, they have investigates and performed the research to find reason behind energy depletion and how can mitigate from it. They have proposed multi metric scheme which was integrated with the existing algorithm OLSR using their routing approach. Authors performed simulation using NS3 environment where they have shown the efficiency in the terms of packet delivery ratio (PDR) which outperform 10$20 \%$ more efficient than the existing standard OLSR technique.

\section{EXISTING ALGORITHM}

The existing algorithm follows the manner in which they are working while transferring the packets. There are following steps which involve in the traditional OLSR technique [1] while transferring the packets.

Step 1: configuring all the nodes and simulation time and other required dimension with the communication participant node.

Step 2: setting up the $\mathrm{X}$ and $\mathrm{Y}$ axis position of each node participating in simulation.

Step 3: setting up the packet size 512 and rate as $20 \mathrm{~kb}$.

Step 4: Put forward the communication in between source and destination node and finally observed the parameter into trace file.

Step 5: plotting the trace files and stop the simulation using traditional OLSR.

\section{PROBLEM DEFINITION}

In the paper upon discussing and observing the recent work we have further noticed that the work done with the low packet numbers and not with the high traffic and weight given onto the nodes and thus the observation can't be monitor according to those steps. In the further work we have observed some problem definitions- 
1. Optimization in weight observation and depletion is not performed.

2. Feasible path monitoring is required to observe.

3. Some other parameter than PDR should be monitor to claim more accuracy of work such as packet loss and optimal energy after communication.

\section{PROPOSED WORK}

In order to define and derive our proposed work we propose a new algorithm which is MET OLSR (Modified Energy Threshold based OLSR) technique, in which a transmission packet modification is considered with optimization which says about the weight assigning on each node and then keep track of weight depletion using TC packet[6], where in tradition OLSR there is no provision of assigning weight and further on TC packet doesn't take care of monitoring and storing it into its component, hence no optimization is performed in existing. Where as in the proposed algorithm we have weight assignment and monitoring policies.

The proposed algorithm depict a enhance feature which includes wormhole attack [11] which occur in network layer. The depicted attack mainly imposed the network jam by presenting the ambiguity in between the source and destination node.

This type of attack usually makes generation of incorrect information in the network such as incorrect traffic generation, message bombing and other sort of DOS attack. It also cause incorrect traffic relaying. Thus our proposed work includes the remedy over the attack cause in the network as a security resistance protocol.

The proposed algorithm steps MET OLSR (Modified Energy Threshold based OLSR) technique:

\subsection{MET Algorithm}

Input: Number of nodes, protocol, energy threshold, trust, energy

Output: Optimal path finding, energy optimization, packet delivery, packet loss.

Steps - Begin

For each node $(\mathrm{i}=0, \mathrm{i}<\mathrm{n} ; \mathrm{i}++)$

\section{\{}

Initialize node I;

Setup node energy : i.e 80,90,100.

Setup node trust : i.e. 1 for each node.

\}reate routing table()

\{

for $\mathrm{i} \leftarrow 1$, NumberofNeighbors

do : Add New Entry in the Routing Table

lookup the all neighbor node and update routing list.

end for $\}$

setup threshold $\$ \mathrm{cr}=80$;

findpath()

\{

For each neighbor to destination;
If $\operatorname{energy}(n)>=c r$

\{

Add to path;

\}else continue lookup alternate path;

Return path; return energy left;

Compute results;

\}End

\section{EXPERIMENT SETUP AND RESULT ANALYSIS}

In order to evaluate the efficiency of our work we have setup an simulation on Machine Red Hat Linux, where we have installed and configured correctly all the patches of NS2 which is a simulator for the network scenario and protocol. We further configured latest OLSR 1 patch in NS2 and performed simulation and result evaluation between existing and proposed protocol.

Table 1: simulation parameter setup environment.

\begin{tabular}{|l|l|}
\hline Simulation Environment setup & Value \\
\hline Dimension & $1500 \mathrm{~m}^{*} 1500 \mathrm{~m}$ \\
\hline Nodes & 30 \\
\hline Traffic type & CBR/UDP \\
\hline Initial node energy & $80-100$ Joules \\
\hline Packet size & 512 bytes \\
\hline Traffic Sources & 3 \\
\hline
\end{tabular}

The Observed simulation parameters: Packet Delivery Ratio (PDR) (in \%): the ratio of the number of packets delivered to the destination nodes over the number of packets sent by the source nodes. We use the normalized PDR, which is defined as the number of packets delivered divided by the number of packets that should ideally been transmitted in this data rate. This gives more representative results.

\section{RESULT ANALYSIS}

\subsection{Comparison of the PDR (packet delivery ratio) over different mobility speed}

Comparison of the PDR for the different speed is presented, in this section. A statistical comparison of the PDR is presented in Table 2. That table contains numerical value of PDR for the different Mobility Speed. That comparison shows, proposed technique provides better results as compare to the existing technique.

A graphical comparison for the various technique is presented in Figure 1, that comparison shows proposed technique provides better results as compare to the existing technique. 
Table 2: comparison of the PDR

\begin{tabular}{|c|c|c|}
\hline Mobility Speed & Existing Technique & $\begin{array}{c}\text { Proposed } \\
\text { Technique }\end{array}$ \\
\hline 0 & 82.77 & 85.93 \\
\hline 1 & 81.54 & 86.54 \\
\hline 5 & 75.27 & 79.6 \\
\hline 10 & 73.19 & 73.89 \\
\hline 15 & 72.49 & 74.33 \\
\hline 20 & 69.59 & 74.49 \\
\hline
\end{tabular}

\section{PDR Vs Mobility Speed}

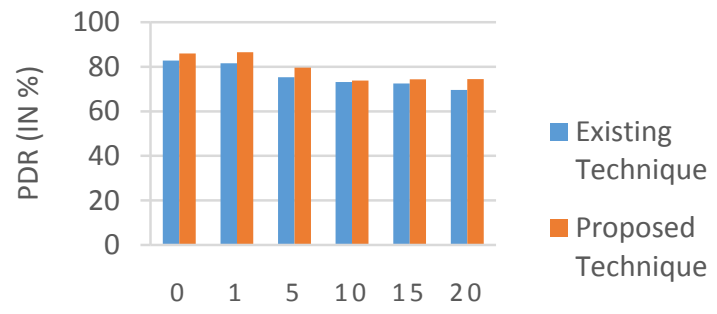

Speed $(\mathrm{m} / \mathrm{s})$

Fig. 1: Comparison of the PDR over mobility Speed.

\subsection{Comparison of the NRL (Normalization \\ Route Load) for existing and proposed technique over mobility speed:}

A comparison analysis of the Normalization Route Load is presented in this section. That comparative analysis shows that proposed technique provides better results as compare to the existing technique. A statistical analysis of the results is presented in Table 3. That shows a numerical comparison of the existing and proposed technique. That comparison shows proposed technique generate less amount of normalization load as compare to the existing technique.

Table 3: a statistical comparison for the NRL (Normalization Route Load) vs Mobility Speed.

\begin{tabular}{|c|c|c|}
\hline Speed & $\begin{array}{c}\text { Existing } \\
\text { technique }\end{array}$ & $\begin{array}{c}\text { Proposed } \\
\text { technique }\end{array}$ \\
\hline 0 & 0.46 & 0.37 \\
\hline 1 & 0.37 & 0.33 \\
\hline 5 & 0.89 & 0.78 \\
\hline 10 & 3.99 & 3.11 \\
\hline 15 & 6.02 & 6.16 \\
\hline 20 & 4.03 & 3.99 \\
\hline
\end{tabular}

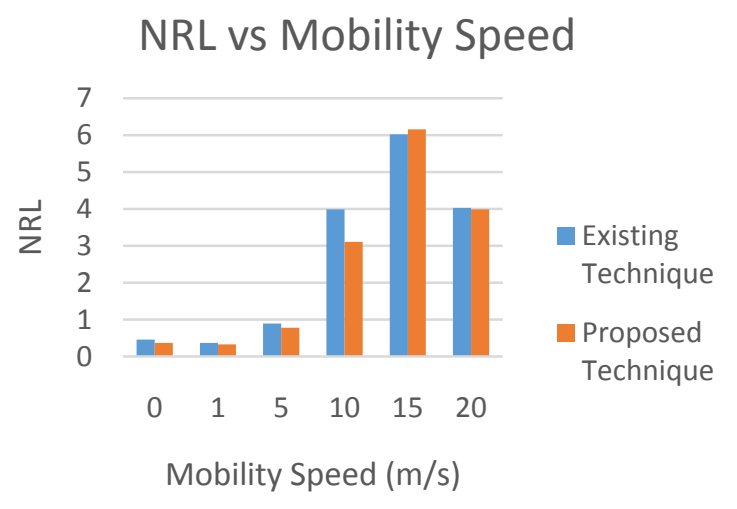

Fig. 2: Comparison of the Normalization load vs mobility speed.

A graphical comparison for the existing and proposed technique is presented in Figure 2. That comparative analysis shows, proposed technique provides better normalization load as compare to the existing technique.

\subsection{Comparison of the end to end delay for the existing and proposed technique over mobility speed:}

Table 4: Comparison of the End to End delay vs mobility Speed.

\begin{tabular}{|c|c|c|}
\hline Speed & $\begin{array}{c}\text { Existing } \\
\text { technique }\end{array}$ & $\begin{array}{c}\text { Proposed } \\
\text { technique }\end{array}$ \\
\hline 0 & 29.2 & 25.41 \\
\hline 1 & 20.84 & 19.42 \\
\hline 5 & 24.95 & 23.15 \\
\hline 10 & 45.12 & 41.17 \\
\hline 15 & 56.20 & 55.65 \\
\hline 20 & 69.96 & 63.03 \\
\hline
\end{tabular}

A comparative analysis over the end to end delay is presented in this section. A statistical analysis for the end to end delay generated by the different techniques is presented in Table 4 . That comparative analysis shows proposed technique less amount of delay as compare to the existing technique.

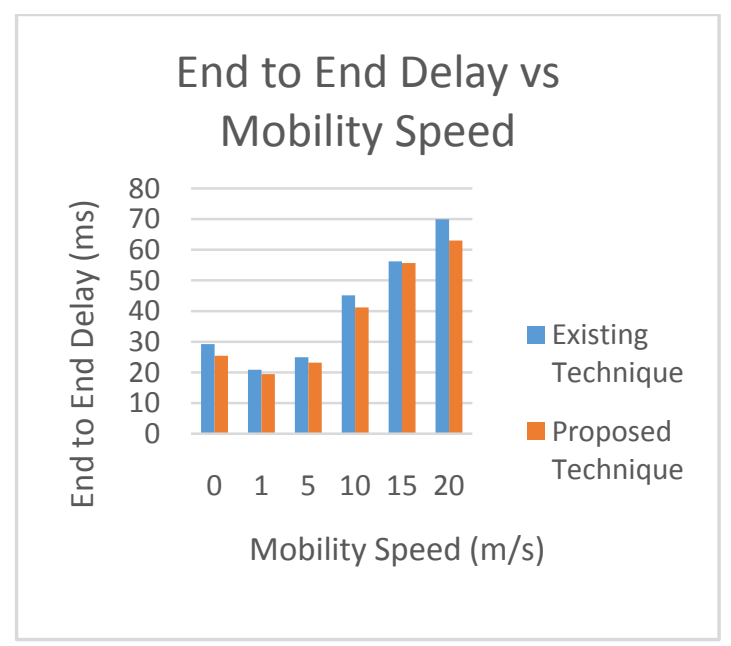

Fig. 3: Comparison of end To end delay vs mobility Speed. 
A graphical comparative analysis for the end to end delay for existing and proposed technique is presented in Figure 3. That comparative analysis shows, proposed technique generate less amount of delay as compare to the existing technique. And provide enhanced technique to route packet in the wireless sensor network scenario.

\subsection{Comparison of the throughput for existing and proposed technique}

A comparative analysis for the existing and proposed technique is presented in this section. A statistical comparison of throughput generated by existing and proposed technique is presented in Table 5. That comparative analysis shows proposed technique provides better throughput as compare to the existing technique. Value for the throughput is presented, in the table 5. That shows proposed technique throughput is better than the existing technique.

Table 5: Comparison of throughput generated by the existing and proposed technique.

\begin{tabular}{|c|c|c|}
\hline Speed & $\begin{array}{c}\text { Existing } \\
\text { Technique }\end{array}$ & $\begin{array}{c}\text { Proposed } \\
\text { Technique }\end{array}$ \\
\hline 0 & 76.84 & 78.46 \\
\hline 1 & 77.27 & 80 \\
\hline 5 & 75.26 & 75.6 \\
\hline 10 & 70.32 & 71.63 \\
\hline 15 & 67.47 & 69.44 \\
\hline 20 & 61.28 & 62.81 \\
\hline
\end{tabular}

\section{Throughput vs Mobility Speed}

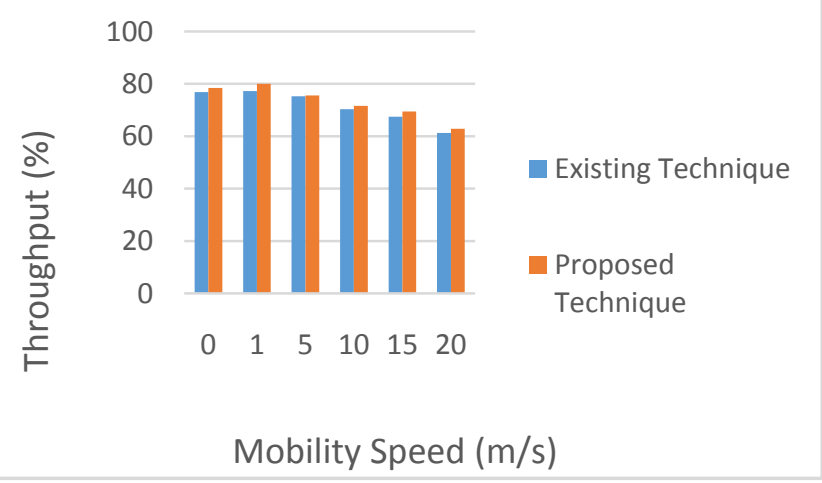

Fig .4: Comparison of throughput vs mobility speed.

A graphical comparison of the throughput for existing and proposed technique is presented in Figure 4. That shows proposed technique generate high throughput as compare to the existing technique.

\section{CONCLUSION}

Wireless sensor network is an area of computation where different energy efficient technique and protocol is proposed. In our work the working module is implemented using NS2 Red hat Linux platform where energy efficient technique is implemented and based on the set threshold energy value optimal path is computed. Thus the observed results using the trace file were generated and it is proven that with the help of energy efficient technique the work can further be concluded using proposed MET technique which give low packet loss i.e. efficient energy management and high PDR (packet delivery ratio) in the network. For future work an enhanced clustering and classification technique can be used to provide optimized performance to route packets from source to destination. That can be reduce the overhead to deal individual node to route packets and provide homogeneous environment to deliver data form source to destination.

\section{REFERENCES}

[1]. Evripidis Paraskevas, Kyriakos Manousakis, Subir Dasand John S. Baras in paper "Multi-Metric Energy Efficient Routing in Mobile Ad-Hoc Networks"2014 IEEE Military Communications Conference.

[2]. Joydeep Banerjee \& Mrinal Kanti Naskar, Utpal Biswas in paper "Leader Selection in Wireless Sensor Networks An Energy Efficient Approach" 2014 International Conference on Control, Instrumentation, Energy, and, Communication (CIEC)

[3]. Kuldeep Vats, Monika Sachdeva, Dr. Krishan Saluja, Amit Rathee in paper "Simulation and Performance Analysis of OLSR Routing Protocol Using OPNET" 2012, IJARCSSE.

[4]. Rahul C. Shah and Jan M. Rabaey" Energy Aware Routing for Low Energy Ad Hoc Sensor Networks"F29601-99- 1- 0169 entitled, "Communication/Computation Pico nodes for Sensor Networks".

[5]. Sonali Sen Baidya and Abhishek Baidya "Energy Conservation in a wireless Sensor Network by an efficient Routing Mechanism" 2015 International conference on communication, Information \& Computing Technology.

[6]. Charu Gupta and Pankaj Sharma "Implementation of Energy Aware Routing Protocol for Mobile Ad Hoc Networks" 2013, IJARCSSE.

[7]. YI Gao \& Wei Dong "COPE: Improving Energy Efficiency with Coded Preambles in Low Power Sensor Networks" 2015, IEEE Transaction and Industrial Informatics.

[8]. Nguyen Duy Tan and Nguyen Dinh Viet "Sleep scheduled and Tree -Based Clustering Routing Protocol for Energy -Efficient in Wireless Sensor Networks" 2015, International Conference on Computing \& Communication Technologies.

[9]. N. Kumar and Dr. C. Suresh Gnana Dhass "Power Aware Routing Protocols in Mobile Adhoc Networks, 2012 IJARCSSE.

[10]. Shital Vaibhavraj gharge and Srinu Dharavath "Virtualization of Wireless Sensor Network with Efficient Power Saving Scheme" 2015, International Conference on Communication, Information \& Computing Technology(ICCICT).

[11].D. Sylvia and Jeevaa Katiravan "Trust based Routing in Wireless Ad Hoc Networks under Adverse Environment" International Journal of Computer Applications (0975 8887)Volume 136 - No.10, February 2016. 